\title{
Proyecciones de la teoría sociológica en América Latina: descripción, análisis y diagnóstico de la modernidad
}

\author{
José Maurício Domingues ${ }^{1}$ \\ IESP-UERJ, Rio de Janeiro, Brasil. \\ Email: jmdomingues@iesp.uerj.br
}

\begin{abstract}
Resumen: ${ }^{2}$ Este artículo examina de manera breve algunos de los debates teóricos más recientessobre América Latinaque, si no siempre están referidos a la sociología o a las ciencias sociales, forman parte de una problemática cuyo examen puede ayudarnos a delinear algunos de los retos y desafíos que la presente situación plantea a estas disciplinas. Para ello, en primer lugar, se abordan las teorías de la "colonialidad del poder” y las teorías “descoloniales”; a continuación se discuten algunas teorías sobre temas y áreas más específicos, procurando dar cuenta de los déficits teóricos que caracterizan el escenario contemporáneo. En ese marco, se sugerirán algunas líneas de desarrollo teórico posibles para la sociología, con énfasis en la modernidad global y los “conceptos-tendencia”, desde hace tiempo ausentes de la teorización latino-americana.
\end{abstract}

Palabras clave: colonialidad del poder, conceptos-tendencia, extroversión

\section{Projections of sociological theory in Latin America: description, analysis and diagnosis of modernity}

\begin{abstract}
This article briefly reviews some of the more recent debates on Latin America, which, that if they do not alwaysrefer to sociology or social sciences, they are part of a problematic whose examination can help us in outlining some challenges that the present situationpresents to these disciplines. For that, we first discuss the theories of 'coloniality of power' and 'de-colonialist' theories; then, somemore specific theories andthemes are identified, aiming t point out theoretical deficitson the contemporary scenery. On this frame, possible trends for theoretical developments for sociology are brought out, with emphasis on global modernity and trend-concepts, for a long time absent from Latin American sociology.
\end{abstract}

Keywords: coloniality of power, concepts-trend, extroversion

\section{Projeções da teoria sociológica na América Latina: descrição, análise e diagnóstico da modernidade}

Resumo: Este artigo examina brevemente alguns dos mais recentes debates teóricos sobre a América Latina, ainda que nem sempre estejam relacionados com a sociologia ou as ciências sociais, eles fazem parte de uma problemática cuja análise 
pode ajudar a delinear alguns dos desafios que esta situação representa para estas disciplinas. Para fazer isso, em primeiro lugar, abordaremos as teorias da "colonialidade do poder" e as teorias "descoloniais"; em seguida, são discutidas algumas teorias sobre temas e áreas mais específicas, tentando explicar os déficits teóricos que caracterizam a cena contemporânea. Neste contexto, se sugerem algumas linhas de desenvolvimento teórico possíveis para a sociologia, com ênfase na modernidade global e os "conceitos-tendência", desde algum tempo ausentesna teorização latino-americana.

Palavras-chave: colonialidade do poder, conceitos-tendência, extroversão.

\section{De la historia al presente}

Las ciencias sociales en América Latina disfrutan de una larga historia yde logros importantes. Esa historia reconoce un punto de inflexión fundamental en las discusiones en torno al pasaje del “ensayismo”a la“sociología profesional”o “científica”, movilizadas en buena medida porFernandes (1958) y Germani (1959) (quienes no dejaron de valorar las ideas, muchas veces seminales, de aquellos primeros estudios). En aquel momento, en efecto, se consolidó una mirada más sistemáticamente sociológica / sobre nuestros países, que acordaba un lugar destacado a la sociología política. También fue entonces que pudo articularse, quizás por primera vez, un espacio de comunicación verdaderamente (sub)continental, con varios temas y problemas delineandouna agendaen buena medida compartida. A los debates metodológicos de largo alcance, se sumaron elaboraciones más substantivas:las teorías de la "marginalidad” y su crítica, el ascenso y difusión de la teoría del "populismo", las tesis sobre el "colonialismo interno”, las discusiones sobre el “desarrollo” y las teorías de la “dependencia”. Cada una, a su modo,se propuso encarar y analizar problemas ciertamente relevantes y urgentes de la realidad regional, e hicieron, en los mejores casos, un aporte original que no podía dejar de reformular las teorías europeas que, de una forma u otra forma, subyacían a sus interpretaciones, al tiempo queponían en juegouna mirada de largo plazo sobre el despliegue de aquellos procesos. Ahora bien, la irrupción de las dictaduras militares en varios países latinoamericanos desarticuló en buena medida, sobre todo después de 1973, este entramado académico e intelectual. En ese marco, fue sólo a partir de los años 1980 que comenzó una recuperación de las ciencias sociales, motorizada en principio por una ciencia política que, bajo la influencia de los estudios doctorales que algunos de sus representantes más reconocidos realizaron en los Estados Unidos (Domingues, 2005), asumió un perfil más específico disciplinariamente.

Desde entonces, poco a poco, un espacio más amplio de discusiones e intercambios fue siendo recuperado. En la promoción de esos lazos subcontinentales, además de los esfuerzos de entidades académicas como Clacso, fue ciertamente gravitante la acción de los movimientos sociales. Sin embargo, preciso es advertir que, salvo algunas pocas excepciones (Sader, 2009; Domingues, [2008] 2009),el recorte o la escala nacional se 
consolidócomo el foco privilegiado de los análisis, incluso en los debates más amplios. Se afirmó, asimismo, una orientación en la que, al tiempo que predominan los estudios de caso, es posible constatar un marcado déficit de teorización -que permanece vigente en la actualidad-, sea en los estudios centrados en un país, sea en aquellos que buscan un dialogo subcontinental. Como contrapartida, las teorías europeas y estadounidenses, con los efectos distorsivos que su incorporación acrítica puede conllevar, adquirieron un renovado peso.

En este artículo me propongoanalizarde manera breve algunos debates recientes sobre América Latina que, si no siempre están referidos a la sociología o a las ciencias sociales, forman parte de una problemática cuyo examen puede ayudarnos a delinear algunos de los retos y desafíos que la presente situación plantea a estas disciplinas. Para ello, en primer lugar, abordaremos las teorías de la "colonialidad del poder" y las teorías “descoloniales”. En segundo lugar, serán discutidas con brevedad algunas de las teorías sobre temas y áreas específicas, procurandoidentificarlos déficitsya señalados. Luego, plantearemos algunas de las líneas de desarrollo teórico posibles para la sociología. En relación con lo primero, sin desconocer la importancia del problema del eurocentrismo, propondremos una sociología global crítica, de carizlatinoamericano, pero realmente de alcancemundial. En relación con lo segundo,llamaremos la atención sobre la necesaria superación de las visiones descriptivas (e incluso analíticas) cuando se muestran incapaces de plantear desarrollos de largo plazo. La historia de las ciencias sociales en los siglos XIX y XX estuvo marcada por la identificación de las tendencias de desarrollo de la modernidad. Hoy, en contraste,parece que hemos desistido de esa búsqueda, tal vez porlas dificultades reales que esa empresa conlleva, tal vez por la presencia de un posmodernismo y un empirismo que rehúyenlos vuelos teóricos más amplios. Si es siempre necesario evitar las miradas demasiado abarcadoras, que definen totalidades excesivamente articuladas y cerradas, no menos imperioso es volver a pensar los procesos de largo plazo y sus direccionalidades. Cabe aclarar, finalmente, que en esta oportunidad, aun cuando se haga mención a los debates globales, limitaré mis referencias a los debates latinoamericanos, dado que, en los límites del presente texto, sería imposible contemplar el conjunto de interlocutores que una discusión más amplia impondría.

\section{Teorías en curso}

El debate más intenso sobre América Latina, que se diseminó por muchas áreas del pensamiento y el quehacer intelectual, tuvo lugar en los últimos años a partir de los trabajos de Aníbal Quijano y su tesis sobre la “colonialidad del poder”, retomada tiempo después por Walter Mignolo y su decidido llamado a una“descolonización”. Referencia de larga data para las ciencias sociales de la región, Quijano (1989) afirmó que la finalización del período colonial no supuso la superación de las maneras de mirar y pensar que reproducen la posición subordinada y eurocéntrica impuesta en 
aquel período. Desarrollada a partirdel siglo XVI, la modernidad es, según este autor, de modo constitutivo y, en tanto, tal "colonialidad", es decir, opresión. La cuestión indígena y su profunda y persistente subordinación constituyen tan sólo la punta de iceberg de un problema mucho mayor cuya resolución exigiría, de un lado, la transformación de las epistemologías con las que estructuramos nuestro pensamiento y del otro, y en términos más sociales, la ampliación de la nación y la ciudadanía. Por su parte, Mignolo (2000 y 2005), partiendo en buena medida de ahí, pero radicalizando el argumento, sostiene la imperiosa necesidad de producir un rechazo completo de la modernidad a partir de un "pensamiento fronterizo" propio, que está dentro y fuera de la modernidad, y que tiene en los indígenas y sus tradiciones sus agentes fundamentales(aun cuando el autor no deje de tener en cuenta a otros tipos sociales en su discusión sobre la "liberación”). De esa forma, el pensamiento “descolonial” podría crecer y ganar presencia, desechando, por lo demás, la sociología en tanto disciplina fundamentalmente moderna.

En un trabajo anterior (Domingues, 2009) examiné esta corriente, en particular los escritos de Mignolo. Baste aquí señalar dos elementos importantes en el marco de la presente discusión. Por un lado, según pude constatar en aquella oportunidad,estos trabajos tienen una visión muy reductiva de la modernidad que desconoce su dimensión emancipatoria para centrarse de forma unilateral en sus elementos opresivos -que, por supuesto, los tiene-. Por el otro, los aportes de esta corriente son poco sociológicos. Lejos de ofrecer una teoría o conjunto de conceptos más o menos sistemáticos, lo que brindanes un punto de vista desde donde denunciar la opresión de las masas populares pero que, construido muchas veces desde una retórica especulativa, no da apoyos analíticos efectivos que puedan servir ala investigación de los científicos sociales.Por supuesto, no es que los problemas planteados no sean importantes. Creo, sin embargo, que otras respuestas son necesarias, en términos de cómo comprendemos el conocimiento y las categorías para el análisis social.

Existen, no obstante, algunos intentos por avanzar en este sentido, intentos quebuscan articular lo que Merton definió en su momento como “teorías de alcance intermedio”. En las últimas décadas, esas iniciativas se concentraron en algunas áreasde investigación: en las teorías de la "sociedad civil” y en debates sobre movimientos sociales, con muchos investigadores, aunque con poca teorización; en la sociología de la cultura, con Jesús Martín-Barbero, Renato Ortiz o Néstor García Canclini (aunque éste prefiera hablar de una tendencia hacia la transición a una "problemática posmoderna”); en la sociología del trabajo, con Enrique de la Garza Toledo como su principal exponente; en demografía, con un grupo numeroso de investigadores cuyo centro se ubica en México; en teoría y sociología del derecho con Colombia comoel centro principal de producción (Domingues, 2005). ${ }^{3}$ Estas teorías, en la medida en que se abren a perspectivas conceptuales más abarcadoras y generalizadoras, tienen la enorme importancia de permitirnos ir más allá de los estudios de casotan frecuentes-como limitados-. 


\begin{abstract}
Ahora bien, pese a esos esfuerzos, la búsqueda de una teoría más general, como ya señalé, es aún una materia pendiente. Aun cuando a veces se encaren ejercicios analíticos y conceptuales más amplios, los límites de los estudios permanecen acotados, dedicados en la mayoría de los casos a un sólo país. Sigue vigente por lo tanto un ostensible déficit teórico que limita el alcance de buena parte de las investigaciones. Ante este escenario, hay quienes, de manera ciertamente excesiva, no dudan en afirmar que el marxismo es -o debería seguir siendo- el marco interpretativo fundamental de la vida social. Si varias de las contribuciones de esta corriente no han perdido su valor, sus pretensiones radicalmente totalizadoras y exclusivistas, así como susdesfasados diagnósticos y estrategias de emancipación, imponen, es claro, una profunda revisión.
\end{abstract}

\title{
Teorías por hacer
}

Como es sabido, las teorías sociológicas -y aquellas que terminaron incorporadas al patrimonio de la disciplina- estuvieron siempre preocupadas por el surgimiento ydesarrollo de la modernidad, civilización que se ha proyectadoglobalmente. El pensamiento latinoamericano, en su fase ensayística, no podía dejar de tratar esta cuestión, una vez que, como observo Leopoldo Zéa (1976), estamos estrechamente vinculados a la expansión de occidente. Por supuesto, en este caso la cuestión se planteó de una manera peculiar dado que, a diferencia de lo que ocurría en los países centrales, no nos era dada la posibilidad de afirmar una universalidad sin más, lo que hizo de la reflexión sobre nuestras particularidades una materia ineludible. La pregunta sobre cómo nos insertábamos en la modernidad, desde una posición que sabíamos que no era central sino derivada, se planteó una y otra vez.

Cuando tiempo después la sociología fue institucionalizada y se estableció como una disciplina académica, la cuestión no perdió vigencia, lo mismo ocurriendo en el caso del marxismo, como lo muestra la larga discusión que se dio dentro de los partidos comunistas y otras corrientes socialistas a lo largo y ancho del continente (Aricó, 1987). Desde sus inicios,en efecto, la mejor ciencia social latinoamericana no rehuyó la problemática, cuestionado ya entonces los alcances y naturaleza del proceso de desarrollo latinoamericano. Lo anterior, por supuesto, no impidió la producción de ciertas reificaciones y una persistente tendencia a hipostasiar una modernidad que, según creían algunos, sólo se hallaba plenamente desarrollada en Europa y Estados Unidos. Este fue el caso, de manera general, de las importaciones de la "teoría de la modernización” estadounidense (ver Lipset y Solari, 1967) y del concepto de "populismo”, elaborado inicialmente por Germani (1965). Emparentado con la teoría de la modernización, aunque no ceñido sólo a ella, este concepto influyó de forma más o menos sistemática sobre una enorme gama de investigaciones en el subcontinente, buena parte de las cuales, no obstante, fue incapaz demantener la sutiliza originaria presente en lasprimeras discusiones acerca del peronismo. Esas discusionesarticulaban una serie de elementos hermenéuticos muy ricos e 
interesantesa la hora de pensar la cuestión de la libertad en su concreto desarrollo en la Argentina de mediados del siglo pasado.

En este marco, el eurocentrismo no fue ciertamente infrecuente entre los más diversos autores, incluso entre aquellostan geniales como Sarmiento quien,por primera vez,intentó elaborar una interpretación de amplio alcance de nuestra realidad que no pudo, sin embargo, trascender aquel sesgo, tal como queda expresado en su brutal consigna: "civilización o barbarie”. A veces el reconocimiento de estas dificultades y la búsqueda de posibles soluciones produjeron algunas reacciones exageradas. Así, por ejemplo, ocurrió con Ramos (1965) quien proponíala realización de una "reducción sociológica”, operación intelectual de perfil fenomenológico que, si bien no desconocía el carácter auxiliar de las teorías extranjeras, demandaba un "vaciamiento" de nuestra mente para poder asir de manera inmediata los datos constitutivos de nuestras realidades nacionales. Desechaba, con ello, y tal como Fernandes (1958) denunció en el marco de una encendida polémica, buena parte de la herencia sociológica occidental. Más adelante, en la conclusión, volveré sobre las razones que llevan a una lectura casi metafísica de la modernidad, en la que los contornos concretos de su emergencia y desarrollo en occidente son universalizados de formaapresurada y equivocada. Lo que importaahora es subrayar que, más allá de los errores y la reproduccióna veces inconsciente de estos problemas,fueron muchos los autores -de Mariátegui y Prebisch a Marini,Cardoso yFals Borda, del Germani yFernandesa González Casanova,O’Donnell y García Linera, de Quijano y Ramos a García Canclini, Zavaleta Mercado y Lechner- que produjeron descripciones empíricas y análisis conceptuales de gran valorsobre los rasgos, al mismo tiempo universales y particulares, de la modernidad latinoamericana.Con todo, y tal como indiqué, ello no significó el desarrollo o la afirmación de una teoría más general, teoría que, por lo demás, para buena parte de esos autores estaba ya dada, sea en el marxismo, sea en el weberianismo o el funcionalismo.

Desde luego, no sería posible realizar un análisis profundo y completo de todo este amplio conjunto de autores, ni tampoco delos problemas que enfrentaron. Baste señalar que las teorías de la dependencia fueron, de manera general, el esfuerzo más abarcador y universalizante producido por las ciencias sociales latinoamericanas. Como sea, lo que quiero sugerir aquí es la imperiosa necesidad de desarrollar una nueva teoría de la modernidad global que, con una mirada emancipatoria y crítica, sea capaz de recoger la vasta herencia teórica europea y estadounidense, así como latinoamericana y de otros países periféricos y semiperiféricos(como Rusia e India)con el fin de incluir, en el seno de una misma articulación conceptual,los elementos universales y particulares que caracterizan los giros y procesos de modernización que se vienen dando en todo el planeta. Semejante empresa, nos daría, según mi opinión, los elementos indispensables pararesponder en mejores condiciones no sólo aquellas cuestiones que el pensamiento latinoamericano y sus despliegues socio-científicos se plantearon una y otra vez, sino también para afrontar los retos y desafíos colocadas por los aportes descoloniales y poscoloniales, a partir de soluciones que, en términos 
teóricos, resulten muchomás adecuadas. De esa forma, también se podría discutir con la muy interesante, pero también limitada, teoría de las “modernidades múltiples” de Shmuel Eisenstadt. A continuación, presento de forma resumida algunos de los argumentos desplegados en trabajos anteriores donde intenté desarrollar los primeros elementos de esta teoría (véase sobretodo Domingues, [2008] 2009 y 2012).

En principio, cabe señalar que la modernidad es un fenómeno ineludiblemente global y multidimensional que, a pesar de ser incapaz en sí misma de cumplir plenamente sus promesas emancipadoras, mantiene el potencial de movilizar a los individuos y las colectividades para realizar los valores progresistas que, de manera variada, son centrales en su imaginario. Aun cuando puedan observarse procesos de intercambio fuertemente entrelazados entre las colonias y los países metropolitanos en su periodo formativo, es preciso reconocer que la modernidad tiene un origen europeo: es en Europa, en efecto, que se forman su imaginario einstituciones fundamentales, y es desde allí que, luego, se globalizan ydesembarcan, desde afuera, enla periferia en proceso de formación. Ese imaginario se fundaba en una concepción ciertamente estrecha de la "racionalidad", dominada por la dimensión instrumental, algo que, en términos ideológicos, le permitió reclamar para sí la capacidad y la responsabilidad de "civilizar” a los otros pueblos del planeta, subordinándolos. Sin embargo, ese imaginario reposaba también sobre la idea, mucha veces reprimida, de que la libertad debía ser concebida de manera igualitaria, en otras palabras, que todos los miembros de la humanidadson sus sujetos y tienen derecho a ella (si bien enlas versiones más duras del racismo se haya buscado dividir la especie según criterios de base biológicapara negarle a una parte de los seres humanosla capacidad racional para el ejercicio de la libertad). Entre los elementos que caracterizan a la modernidad globalizada y que le dan su carácter multidimensional, figuran el estado moderno racional-legal y burocrático (que de hecho, si bien no de derecho, asume a veces un perfil patrimonialista), la ciudadanía, la democracia representativa y la esfera pública, el capitalismo y la mercantilización universal de la vida social, el patriarcado moderno individualista y el racismo institucionalizado, los procesos de individualización y de apertura de las identidades individuales y colectivas.En ese marco de prácticas, elementos imaginarios e instituciones, la libertad, como valor, es a veces afirmada, aunque con alcance limitado, y a veces negada de forma explícita o tácita. Los movimientos socialistas, comunistas y anarquistasintentaron abrir la modernidad a una posmodernización en la que se realizaran por fin sus promesas, pero hasta hoy, como es sabido, no lograron el éxito esperado.

Ahora bien, en la medida en que la expansión de la modernidad conllevó su hibridización (sintética y sincrética) con elementos provenientes de otras civilizaciones, es preciso avanzar con el argumento. Fruto de su difusión, en efecto, la modernidad se conformó como una civilización global heterogénea, en la cual no podían dejar de mezclarse o combinarse elementos que, si bien modernizados,provenían de otras civilizaciones. Esto, por supuesto, no ocurrió de manera automática ni tampoco unidireccional. 
Fueron giros modernizadores específicos, impulsados por individuos y colectividades múltiples (o "subjetividades colectivas": movimientos sociales, clases, géneros, "razas", grupos étnicos, movimientos y organizaciones religiosas, familias, empresas, estados, etc.), los que tejieronla modernidad global híbrida tal cual la conocemos, en el marco de un proceso contingente en el que, de todos modos, es posible reconocerde modo empírico un vector principal, difundidopor la imposición más o menos violenta o por la seducción que producía. Si los sistemas de dominación son, como indiqué,aspectos fundamentales de la modernidad, los valores emancipatorios -sobre todo la libertad igualitaria, que implica también otras formas de solidaridad- mantienen, cabe insistir, su fuerza (a pesar de que el momento actual no sea el más propicio para los movimientos que los sostienen y promueven). ${ }^{4}$

Dicho lo anterior, se hace necesario dar un paso más pues, hasta aquí, a pesar de haber introducido un conjunto variado de categorías de análisis, corremos el riesgo de permanecer en un plano meramentedescriptivo de la vida social, con una aprehensión limitada de su dinámica y movimiento. Hasta no hace mucho, como ya adelantamos, una de las labores fundamentales de las ciencias sociales era la búsqueda e identificación de las direcciones de desarrollo que caracterizaban y definían a las "sociedades. Si había quienes se preocupaban por las tendencias vinculadas al capitalismo (y su tendencia a la expropiación general y la formación de un proletariado mayoritario y revolucionario, la concentración y centralización del capital, el cambio de su composición orgánica y la baja tendencial de la tasa de ganancia, etc.) y había quienes preferían preocuparse por aquellas vinculadas a la racionalización de las diversas esferas de valor (de sus imaginarios, prácticas, y formas o estilos de vida, en procesos multidimensionales pero articulados), la búsqueda de esas tendencias era moneda corriente entre los cientistas y autores de las más diversas orientaciones. América Latina no fue, en este sentido,una excepción. Cabe mencionar aquí, a modo de ejemplo, aGermani (1965) y su análisis de la transición de las sociedades "adscriptivas" a las modernas (proceso en el que, cabe notar, surge el "populismo"), a Fernandes (1975) y su preocupación por lacapacidad de despliegue del imaginario liberal en el proceso (revolucionario) de independencia latinoamericano -con referencia específica a Brasil-, a Nun (2001) y Quijano (1998) y su discusión sobre la existencia de una "masa" o "polo" marginal en las economías y sociedades de América Latina, vinculada a su inserción y funciones específicas, dependientes, dentro del cuadro más general del capitalismo global. Ahora bien, en la actualidad, tanto en las ciencias sociales globales como en las latinoamericanas, poco quedó de este tipo de preocupaciones y búsquedas. Hay,por supuesto, excepciones, como la que, por ejemplo, se puede encontrar en ciertos análisis más recientes de los procesos de individualización y cambio en los vínculos sociales en Chile y América Latina (Araujo y Martucelli, 2012). Como sea, esas excepciones son raras. Y, sin embargo, es justamente este tipo de perspectivala que, según creo,es necesario retomar con decisión si queremos, por un lado, plantearnos un trabajo capaz de identificar y reconstruir de manera analítica la dinámica de la vida social moderna y, por el otro, rechazar una 
visión estática y ahistórica de la modernidad, como si esta particular civilización fuese el horizonte último del desarrollo de la humanidad.

En este sentido, lo que propongo es volver a discutir las tendencias de desarrollo de la modernidad: las tendencias que la engendraron, las que la reproducen de manera duradera y continua, y las que nos pueden llevar, teniendo siempre en cuenta su carácter híbrido, más allá de su imaginario, prácticas e instituciones. Para eso, es necesario volver a poner el foco de nuestra mirada en aquello que se puede definir como conceptos-tendencia (Domingues, 2014), herramientas de análisis capaces de conceptualizar la formación de las instituciones modernas y su imaginario -instituciones que ya fueron identificadas por la sociología, aunque su caracterización precisa generesiempre controversias- ${ }^{5}$ En este marco, los mecanismos, teóricamente definidos,mediante los cuales la modernidad se origina, se cristaliza como civilización y se despliega(con permanencias y cambios), así como la preocupación por su posible superación -en dirección emancipadora o notienen que estar en el centro de nuestros esfuerzos sociológicos (algo que, en mi opinión,debería hacerse a partir de una concepción analítico-realista del mundo). Fundados en la articulación de consecuencias intencionales y no intencionales de la acción individual y del movimiento colectivo, los mecanismos dan cuenta de regularidades que, sin embargo, no constituyen "leyes" ("naturales" o de "hierro")ni permiten postular la idea de una uniformidad de la naturaleza (social). Lejos de ello,los procesos así concebidos cambian a lo largo del tiempo, sin que podamos, necesariamente, tener control sobre las tendencias que así se conforman. Finalmente, y en relación con lo anterior, es preciso volver a plantearse la indagación sobre la relación entre las diversas tendencias, evitando recaer en el unilateralismopropio del "materialismo histórico" pero también de aquellas corrientes que, aceptando en principio el carácter multimensional de la modernidad, hacen de la "racionalidad" un hilo unificador. Como podrá apreciarse, la tarea planteada no es sencilla. Sin embargo, resulta insoslayable si queremos avanzar en el estudio científico de nuestras sociedades. Semejante iniciativa nos permitiría sin dudas describir la realidad social de una manera más plena y distanciada del sentido común, hoy excesivamente presente en las ciencias sociales; algo que, por otra parte, nos pondría en mejores condiciones a la hora deentablar un diálogo más rico, en términos sociales, intelectuales y políticos, con audiencias o públicos más amplios. También, nos permitiría producir diagnósticos más precisos sobre la modernidad y, elemento particularmente relevante para la teoría crítica, definir posibles estrategias emancipadoras, preocupación central desde Marx en adelante, pero también, y de modo más amplio, en buena parte de la tradición sociológicaen general.

Por supuesto, las tareas teóricas aquí planteadas no son las únicas posibles y necesarias para la sociología latinoamericana y, claro está, para la sociología global. Existen sin dudas una serie de retos particulares en los diversos campos de teorización más específicos, sea en términos substantivos, sea en lo que hace a cuestiones conceptuales más transversales(en el sentido, por ejemplo, de rescatar teorías como la de la dependencia), sea en 
lo que se refiere a las metodologías o a los temas más concretos de investigación, cuya productividad será, sin embargo, tanto mayor cuanto más atentos estén los investigadores a los problemas metodológicos y teóricos más amplios aquí señalados. Ello es obvio en lo que hace a la ya muchas veces revisitada problemática de la "estructura” y la "agencia”, problemática que es sólo válida si es planteada en conexión con los procesos sociales más concretos. Por otra parte, preciso es aclarar aquí que, aunque nuestra labor responda a los cánones de la ciencia moderna, empresa de origen occidental, lo que hemos planteado no niega la posibilidad de aprender con otros tipos de conocimiento y "epistemologías", aun cuandoquienes las promueven se limiten muchas veces a reivindicar su carácter novedoso, sin demostrar de modo más concreto sus características y potencialidades.[6] Para terminar, puede ser útil plantearun tema más general que se relaciona con la formaen que organizamos y producimos el conocimiento.

\section{A modo de conclusión: dependencia, extroversión y autonomía}

Me gustaría reflexionar este último apartado sobre algunas de las razones que hacen que los sociólogos latinoamericanos evitemos, seamos más o menos conscientes de ello,la elaboración de teoríasque la práctica de las ciencias socialesmuchas vecesnos plantea. ¿Por qué somos tan proclives a reproducir, sin más,las teorías provenientes de Europa y Estados Unidos, aun cuando muchas veces esas teorías resulten claramente inadecuadas, tímidaso poco interesantes? En principio, podemos pensar que hay algo en nuestra posición social como intelectuales y científicos sociales, posición definida por la división del trabajo, que hace que nos relacionemos, casi como metafísicos, de un modo más estrecho con las ideas que circulan globalmente que con la vida social que tenemos delante de nosotros. A eso, sin dudas, se suma una situación de dependencia ligada a una particular geopolítica del conocimiento vinculada, por un lado, al control en manos de los centros de los principales circuitos de producción, publicación y circulación de los saberes, y, por el otro, a lasecular apertura de los "campos" culturales periféricos y semiperiféricos, constituidos a partir de una duradera y perenne “extroversión” (Miceli, 1972 [2005], pp.132ss; Domingues, 1992; Beigel, 2011; Bringel y Domingues, 2015).

Si es cierto que la dependencia se produce y reproduce regularmente mediante una serie de mecanismos como los que aquí fueron presentados, también es cierto que siempre es posible oponer otros mecanismos, de debate y articulación intelectual(dentro de nuestros países, en América Latina y más en general en las periferias y semiperiferias) con el fin de contrarrestar los efectos de las desventajas que la geopolítica del conocimiento nos impone. Por supuesto, la situación de dependencia y extroversión afectaa todas las áreas de las ciencias sociales. Sin embargo, es en el campo de la teoría que esta situación es más clara y aguda, en la medida en que a menudo la elaboración teórica aparece como una empresa de gran 
dificultad y prestigio,reservadapor lo tanto para los científicos del norte, únicos capaces de abordar una tarea de carácter universalizante. Avanzar en esta agenda, retomando los esfuerzos más vigorososque, como vimos, en el pasado emprendió la sociología latinoamericana, sería un logro de grandes repercusiones para la sociología y el resto de las ciencias que comparten con ella esta problemática existencia. No se trata, es claro, de un reto trivial o sencillo, pero tampoco de una empresa imposible de poner en marcha. 
Polis, Revista Latinoamericana, Volumen 14, $N^{\circ} 41,2015$

\section{Notas}

${ }^{1} \mathrm{PhD}$ en sociología por la London School of Economics and PoliticalScience, profesor e investigador del IESP-UERJ y autor de, entre otros, los siguientes libros: $L a$ modernidad contemporánea en América Latina (Buenos Aires: Siglo XXI y Clacso, 2009); Desarrollo, periferia y semiperiferia en la tercera fase de la modernidad global (Buenos Aires: Clacso, 2012); y Global Modernity, Development and Contemporary Civilization (Nueva York y Londres: Routledge, 2012).

${ }^{2}$ Agradezco a Juan Pedro Blois los comentarios y la revisión del español.

${ }^{3}$ En Domingues y Maneiro, 2006, se encuentra una selección de esta bibliografía.

${ }^{4}$ Vale notar que se puede periodizar la modernidad, lo que hice en tres fases: liberal restricta/colonial, estatalmente organizada y contemporánea (o de articulación mista).

${ }^{5}$ En qué medida, más allá de lo que se refiere a las instituciones, se puede hablar de tendencias de desarrollo del imaginario, que es necesariamente "magmático" y en principio amorfo (como nos enseñó Castoriadis), es algo que prefiero todavía dejar abierto.

${ }^{6}$ La sociología histórica, si bien más histórica que sociológica, recibió una contribución relevante con el trabajo de Alsandi y Giordano, 2012. 


\section{Bibliografía}

Araújo, Kathya y Martucelli, Danilo (2012), Desafíos comunes. Retrato de la sociedad chilena y sus individuos. Lom. Santiago.

Aricó, José (1978), “O marxismo latino-americano na época da Terceira Internacional”, in Hobsbawn, Eric(org.), História do marxismo, vol. 8. Paz e Terra. Rio de Janeiro.

Beigel, Fernanda (2011), “Dependencia académica”, Diálogo global, vol. 2.

Bringel, Breno e Domingues, José Maurício (2015), “Teoria social, extroversão e autonomia: impasses e horizontes da sociologia (semi)periférica contemporânea”, Cadernos CRH, vol. 28.

Domingues, José Maurício ([1992] 2003), “A América. Intelectuais, interpretações e identidades”, in Do ocidente à modernidade. Intelectuais e mudança social. Civilização Brasileira.Rio de Janeiro.

Ídem (2005), “Sociologíabrasileña, Latinoamérica y latercera fase de lamodernidad”, Estudios sociológicos, vol. XXIII, no. 68.

Ídem (2008 [2009]), La modernidad contemporánea en América Latina. Siglo XXI y Clacso. Buenos Aires.

Ídem (2009), “Global modernization, 'coloniality’ and a criticalsociologyforLatinAmerica”, Theory, Culture \&Society, vol. 26. En portugués:enTeoria crítica e (semi)periferia. 2011. Editora UFMG.Belo Horizonte.

Ídem (2012), Global Modernity, Development, and Contemporary Civilization: towards a Renewal of Critical Theory. Routledge. Nueva York y Londres. En portugués: 2013. Editora UFMG. Belo Horizonte.

Ídem (2014), “Global modernity, levels of analysis and conceptual strategies”, Social Science Information, vol. 53.

Domingues, José Maurício y Maneiro, María (orgs) (2006), América Latina hoje. Civilização Brasileira. Rio de Janeiro.

Fernandes, Florestan (1958), A etnologia e a sociologia no Brasil: ensaios sobre aspectos da formação e do desenvolvimento das ciências sociais na sociedade brasileira. Anhambi. São Paulo.

Ídem (1975), A revolução burguesa no Brasil. Zahar. Rio de Janeiro.

Germani, Gino (1959), Desarrollo y estado actual de la sociología latinoamericana. Cuadernos del Boletín del Instituto de Sociología. Buenos Aires. 
Ídem (1965), Política y sociedad en una época de transición. Paidós. Buenos Aires.

Lipset, Martin S., y Solari, Aldo (orgs.) (1967), Elites in Latin America. OxfordUniversity Press,Nueva York.

Miceli, Sérgio (1972 [2005]), Anoite da madrinha. Cia. das Letras. São Paulo.

Ramos, Guerreiro (1965), A Redução Sociológica: introdução ao estudo da razão sociológica. Edições Tempo Brasileiro (2a edición, corregida y ampliada). Rio de Janeiro.

Mignolo, Walter D. (2000), Local Histories/Global Designs: Coloniality, Subaltern Knowledges, and Border Thinking. Princeton University Press. Princeton, NJ.

Ídem (2005), The Idea of Latin America. Blackwell. Oxford.

Nun, José (2001), Marginalidad y exclusión social. Fondo de Cultura Económica. Buenos Aires y México.

Quijano, Aníbal (1989), “Coloniality and modernity/rationality”, Cultural Studies, vol. 21.

Ídem (1998), La economía popular y sus caminos en América Latina. Mosca Azul. Lima.

Sader, Emir (2009), El nuevo topo. Los caminos de la izquierda latinoamericana. Siglo XXI y Clacso. Buenos Aires.

Zéa, Leopoldo (1976), El pensamiento latinoamericano. Ariel. Barcelona.

Recibido: 17.06.2015

Aceptado: 15.08.2014 